\title{
CUT-AND-PASTE DEFORMATIONS OF RIEMANN SURFACES
}

\author{
Scott A. Wolpert*
}

Cut-and-paste provides a simple procedure for constructing compact Riemann surfaces. The method also can be used to construct holomorphic families. We shall examine three such constructions: the Schiffer variation, pinching a collar, and plumbing a node.

There is a simple procedure for computing the infinitesimal variation of a cut-and-paste family. The method comes from the Kodaira-Spencer approach to deformation theory. The key point is to record the relative sliding of the open sets, which are overlapped to form the surface. In Section 1 we sketch the procedure for compact Riemann surfaces. The succeeding sections are devoted to analyzing the three specific constructions.

The standard construction for opening a node is plumbing, giving a 1-parameter family. Our main result is a formula (Lemma and Corollary, Section 4) comparing two plumbings of a given node. A simple consequence is the observation that the general 1-parameter deformation for opening nodes is not given by a plumbing. In fact if $R$ is a Riemann surface with a single node, then there is a three dimensional subspace $\mathcal{S}$ of the space of infinitesimal (stable curve) deformations of $R$, such that the initial tangent of a plumbing lies in $\mathcal{S}$.

The author would like to thank David Eisenbud, Joe Harris and Daniel Swearingen for their advice and suggestions. The author would also like to thank the Institute for Advanced Study for its support and hospitality.

\section{Preliminaries}

We start with a sketch of the Kodaira-Spencer approach for deformations of a Riemann surface. References are Morrow-Kodaira, [MwK, Chapters 1 and 2] and Gunning, Lectures on Riemann surfaces, [G, Chapters 1 through 7].

Let $R$ be a compact Riemann surface with finite atlas $\left(U_{a}, z_{a}\right)$, i.e. each $z_{a}$ is a biholomorphism from $U_{a} \subset R$ into $\mathbf{C}$. We are interested in various line bundles over $R$. A section $\phi$ of the $R$-canonical bundle $\kappa$ is a collection of $\mathbf{C}$-functions $\left\{\phi_{a}\right\}, \phi_{a}$ defined on $U_{a}$, such that $\phi_{a}\left(d z_{a} / d z_{b}\right)=\phi_{b}$ on $U_{a} \cap U_{b}$. Alternately $\phi$ is a differential 1 -form of type $d z$. A second example of a line bundle on $R$ is a

\footnotetext{
* Partially supported by the National Science Foundation.
} 
point-bundle. Let $f$ be a holomorphic function defined on a neighborhood $U$ of a point $p \in R$, and vanishing only at $p$ to order 1 . For simplicity of notation assume that $U=U_{0}$ is an element of the atlas for $R$. A section of the point bundle $\zeta_{p}$ is a pair of $\mathbf{C}$-functions: $\tau_{0}$ with domain $U_{0}$ and $\tau_{1}$ with domain $R-p$, such that $\tau_{1} f=\tau_{0}$ on $U_{0} \cap(R-p)$. More generally a section $\Psi$ of the line bundle $\lambda=\kappa^{q} \otimes \bar{\kappa}^{r} \otimes \zeta_{p}^{s}$ over $R$ is a collection of functions $\left\{\Psi_{a}\right\}, \Psi_{a}$ defined on $U_{a}$, such that:

$$
\Psi_{a}\left(\frac{d z_{a}}{d z_{0}}\right)^{q}\left(\frac{\overline{d z_{a}}}{d z_{0}}\right)^{r} f^{s}=\Psi_{0} \quad \text { on } \quad U_{0} \cap U_{a}
$$

and for $a, b \neq 0$

$$
\Psi_{b}\left(\frac{d z_{b}}{d z_{a}}\right)^{q}\left(\frac{\overline{d z_{b}}}{d z_{a}}\right)^{r}=\Psi_{a} \quad \text { on } \quad U_{a} \cap U_{b} .
$$

The sections of $\lambda$ over $R$ form a $\mathbf{C}$-vector space $\Gamma(R, \lambda)$. And of course we may also consider the sections $\Gamma(V, \lambda)$ over an open subset $V \subset R$. In fact the collection of groups $\Gamma(U, \lambda), U \subset R, U$ open, determine a sheaf, the sheaf of germs of sections of $\lambda$. Two important subsheaves are $\mathcal{E}(\lambda)$ the sheaf of germs of smooth sections (the local representatives $\Psi_{a}$ are smooth) and $\mathcal{O}\left(\kappa^{q} \otimes \zeta_{p}^{s}\right)$ the sheaf of germs of holomorphic sections (the local representatives are holomorphic). Of particular importance for deformations of Riemann surfaces are sections of the line bundles $\kappa^{-1}$ (vector fields), $\kappa^{\otimes 2}$ (quadratic differentials), and $\kappa^{-1} \otimes \bar{\kappa}$ (an $L^{\infty}$ section is a Beltrami differential).

Fix a particular line bundle $\lambda$ and let $\mathcal{S}$ be the associated sheaf of germs of sections. A $k$-cochain $\sigma$ with values in $\mathcal{S}$ is an assignment: to each $(k+1)$-tuple $U_{0}, \ldots, U_{k}$ of sets of the cover $\left\{U_{a}\right\}$ with $U_{0} \cap \ldots \cap U_{k} \neq \emptyset$ is assigned a section $\sigma_{0 \ldots k}$ of $\Gamma\left(U_{0} \cap \ldots \cap U_{k}, \lambda\right)$. The set of $k$-cochains $C^{k}\left(\left\{U_{a}\right\}, \mathcal{S}\right)$ is a $\mathbf{C}$-vector space with a natural coboundary operator $\delta: C^{k}\left(\left\{U_{a}\right\}, \mathcal{S}\right) \rightarrow C^{k+1}\left(\left\{U_{a}\right\}, \mathcal{S}\right)$. We will only consider the simplest case $\delta: C^{0} \rightarrow C^{1}$. A 0 -cochain $\sigma$ is an assignment $U_{a} \mapsto \sigma_{a} \in \Gamma\left(U_{a}, \mathcal{S}\right)$; its coboundary is the 1-cochain $U_{a} \cap U_{b} \mapsto \sigma_{b}-\sigma_{a} \in \Gamma\left(U_{a} \cap\right.$ $\left.U_{b}, \mathcal{S}\right)$. The $k$-cochains $\sigma \in C^{k}$ with $\delta \sigma=0$ form a subgroup, the $k$-cocycles; the image $\delta: C^{k-1} \subset C^{k}$ also forms a subgroup, the $k$-coboundaries. The quotient $k$ cocycles/ $k$-coboundaries is the $k^{\text {th }}$ Cech cohomology group $\breve{H}^{k}\left(\left\{U_{a}\right\}, \mathcal{S}\right)$ relative to the cover $\left\{U_{a}\right\}$. If we require that all nonempty intersections $U_{0} \cap \ldots \cap U_{m}$ be topological discs then the Cech groups are actually independent of the choice of cover. In any case a $k$-cocycle determines a class in $\breve{H}^{k}(R, \mathcal{S})$.

A second cohomology theory is for the $\bar{\partial}$ operator. As a sample case we start with a holomorphic line bundle $\xi$ with transition functions $\xi_{a b}$ ( $\xi_{a b}$ is nonvanishing holomorphic, defined on $U_{a} \cap U_{b}$ with $\xi_{a b} \xi_{b c}=\xi_{a c}$ on $\left.U_{a} \cap U_{b} \cap U_{c}\right)$. A smooth section $\Gamma(R, \mathcal{E}(\xi))$ is an assignment of smooth functions $\phi_{a}$ on $U_{a}$ such that $\phi_{a}=\xi_{a b} \phi_{b}$ on $U_{a} \cap U_{b}$. The collection of functions $\left\{\left(U_{a},\left(\partial \phi_{a} / \partial \bar{z}_{a}\right)\right)\right\}$ transform by the rule 


$$
\frac{\partial \phi_{a}}{\partial \bar{z}_{a}}=\xi_{a b}\left(\frac{\overline{d z_{b}}}{d z_{a}}\right) \frac{\partial \phi_{b}}{\partial \bar{z}_{b}}
$$

note that this is simply the transformation law for sections of $\xi \otimes \bar{\kappa}$. Thus $\bar{\partial}$ maps sections of $\xi$ to sections of $\xi \otimes \bar{\kappa}$. We will only need the Dolbeault group

$$
H_{\bar{\partial}}^{0,1}(R, \mathcal{E}(\xi))=\frac{\Gamma(R, \mathcal{E}(\xi \otimes \bar{\kappa}))}{\bar{\partial} \Gamma(R, \mathcal{E}(\xi))}
$$

$\left(\left(U_{a},\left(\partial \phi_{a} / \partial \bar{z}_{a}\right)\right)\right.$ is an example section of $\left.\xi \otimes \bar{\kappa}\right)$. For $\xi=\kappa^{-1}, \Gamma(R, \mathcal{E}(\xi \otimes \bar{\kappa}))$ is the space of smooth Beltrami differentials and $\Gamma(R, \mathcal{E}(\xi))$ the smooth vector fields. The Dolbeault isomorphism provides that

$$
\check{H}^{1}(R, \mathcal{O}(\xi)) \cong H_{\bar{\partial}}^{0,1}(R, \mathcal{E}(\xi)) .
$$

We would like to recall how to evaluate the isomorphism. It will be enough to consider an open cover of $R$ by two sets: $U, V$ such that $U \cap V$ is an annulus $A$. Assume that a cohomology class $(\sigma) \in \check{H}^{1}$ is represented by the assignment $U \cap$ $V \mapsto \sigma \in \Gamma(U \cap V, \mathcal{O}(\xi))$. Let $\gamma$ be a simple closed curve, representing the core of $A ; \gamma$ separates $A$ into annuli $A_{U}$ adjoining $U$, and $A_{V}$ adjoining $V$. Let $\chi$ be a smooth function on $R$ (an approximate characteristic function of $V$ ) such that $\chi$ is identically 1 on $V-A_{U}$ and 0 on $U-A$.

Definition. A formal potential $F$ for $\sigma$ is the 0 -cochain with values in $\mathcal{E}(\xi)$ given by

$$
F= \begin{cases}\chi \sigma & \text { on } U \\ 0 & \text { on } V-\overline{A_{U}}\end{cases}
$$

The Čech coboundary $\delta F$ (restricted to $A_{V}$ ) is given by the assignment $\left.U \cap\left(V-\overline{A_{U}}\right) \mapsto \sigma\right|_{A_{V}} \in \Gamma\left(A_{V}, \mathcal{O}(\xi)\right)$ (the reader may check that the 1-cocycles $\left.U \cap\left(V-\overline{A_{U}}\right) \mapsto \sigma\right|_{A_{V}} \in \Gamma\left(A_{V}, \mathcal{O}(\xi)\right)$ and $U \cap V \mapsto \sigma \in \Gamma(U \cap V, \mathcal{O}(\xi))$ represent the same class in $\left.\check{H}^{1}(R, \mathcal{O}(\xi))\right)$. Now for the Dolbeault coboundary of $F: \bar{\partial} F$ is a smooth section of $\xi \otimes \bar{\kappa}$ over $U \cup V$ (the ambiguity in the definition of $F$ on $A_{V}$ is holomorphic, thus annihilated by $\left.\bar{\partial}\right)$. Indeed $\bar{\partial} F \in \Gamma(R, \mathcal{E}(\xi \otimes \bar{\kappa}))$ and its class $H_{\bar{\partial}}^{0,1}(R, \mathcal{E}(\xi))$ is the image by the Dolbeault isomorphism of the original $(\sigma) \in \mathscr{H}^{1}(R, \mathcal{O}(\xi))$. In brief: the image by the Dolbeault isomorphism of $(\sigma) \in \check{H}^{1}(R, \mathcal{O}(\xi))$ is the class of $\bar{\partial} F$ in $H_{\bar{\partial}}^{0,1}(R, \mathcal{E}(\xi))$ for $F$ a formal potential for $\sigma$.

There is an obvious question: when does $(\sigma) \in \check{H}^{1}(R, \mathcal{O}(\xi))$ represent the trivial class? By a direct approach this would be a tricky combinatorial matter: does there exist a 0 -cochain with coboundary $\sigma$ ? A simpler approach is to consider the linear functional given by $(\sigma)$, acting on the dual space $\breve{H}^{0}\left(R, \mathcal{O}\left(\kappa \otimes \xi^{-1}\right)\right)$. 
From the Dolbeault isomorphism and the specific form of Serre duality the pairing of $(\sigma)$ and $\phi \in \check{H}^{0}\left(R, \mathcal{O}\left(\kappa \otimes \xi^{-1}\right)\right)$ is simply

$$
((\sigma), \phi)=\int_{R} \bar{\partial} F \phi
$$

where $F$ is a formal potential for $(\sigma)$. Recalling the definition of $F$ we note $\bar{\partial} F=:=0$ on $R-A$ and thus

$$
\int_{R} \bar{\partial} F \phi=\int_{A} \bar{\partial} F \phi=\int_{\partial A_{U}} F \phi=\int_{\gamma \approx \partial U} \sigma \phi
$$

the last equality follows since $F=\chi \sigma$ vanishes on the outer boundary of $A_{U}$ and is 1 on the inner boundary $\gamma$. In brief: the pairing of $(\sigma) \in \check{H}^{1}(R, \mathcal{O}(\xi))$ and $\phi \in \check{H}^{0}\left(R, \mathcal{O}\left(\kappa \otimes \xi^{-1}\right)\right)$ is the period $\int_{\gamma} \sigma \phi$.

We wish to consider deformations of a compact surface $R$ with a finite number of distinguished points $p_{1}, \ldots, p_{n}$. The space of infinitesimal deformations is $\check{H}^{1}\left(R, \mathcal{O}\left(\left(\kappa \zeta_{p_{1}} \cdots \zeta_{p_{n}}\right)^{-1}\right)\right)$ and its dual is $\check{H}^{0}\left(R, \mathcal{O}\left(\kappa^{\otimes 2} \zeta_{p_{1}} \cdots \zeta_{p_{n}}\right)\right)$. We review the actual calculation of the 1-cocycle representing a deformation. Suppose that $R_{t}$ is a family of surfaces given by a cut-and-paste construction with a parameter $t$. It will be enough to consider a simple case, two Riemann surfaces glued by a single map. Let $U$ and $V$ be Riemann surfaces and $f$ a biholomorphism of an open set in $U$ to an open set in $V$. Form the identification space of $U$ and $V$ by the $\operatorname{map} f, U \cup V / \sim_{f}$, i.e for $u \in U, v \in V$ then $u \sim_{f} v$ provided $u \in \operatorname{Domain}(f)$ and $f(u)=v$. The quotient space $U \cup V / \sim_{f}$ is a Riemann surface. In fact if $f$ depends holomorphically on a parameter $t$ then $R_{t}=U \cup V / \sim_{f}$ gives a holomorphic family. The family is constructed as follows: let $D$ be the domain for $t$, define an equivalence relation $\simeq_{f}$ on $(U \cup V) \times D$ generated by $(u, t) \simeq_{f}\left(v, t^{\prime}\right)$, provided that $u \in \operatorname{Domain}(f), f(u, t)=v$ and $t=t^{\prime} ;(U \cup V) \times D / \simeq_{f}$ is fibered over $D$ with fibre $R_{t}$. To compute the infinitesimal variation $d R_{t} / d t$, let $z_{0}$ be a generic coordinate on $U$ and $z_{1}$ a generic coordinate on $V$. Then $f$ is given as $z_{1}=f\left(z_{0}, t\right)$. A geometric description is immediate: for $z_{0}$ fixed, as $t$ varies $f\left(z_{0}, t\right)$ traces out the points in the $z_{1}$-region $V$, that $z_{0}$ will be identified to. And thus the $t$-derivative of $f$ gives the infinitesimal variation of the identification. Specifically the 1-cocycle

$$
\theta(V, U)=\frac{\partial f}{\partial t} \frac{\partial}{\partial z_{1}} \quad \text { on } \quad U \cap V \subset R_{t}=U \cup V / \simeq_{f}
$$

with values in $\mathcal{O}\left(\kappa^{-1}\right)$ represents the infinitesimal deformation. 


\section{The Schiffer variation}

An excellent example of a family obtained by varying a cut-and-paste construction is found in the work of Schiffer. We paraphrase the description from Section 7.8 of $[\mathrm{ScS}]$. Let $\gamma$ be an analytic Jordan curve in a surface $R$. Suppose for simplicity that $\gamma$ lies in the domain of a local coordinate $z_{1}$. Let $r\left(z_{1}\right)$ be a function which is analytic in a neighborhood of $\gamma$. For $t$ sufficiently small the function $f\left(z_{1}, t\right)=z_{1}+\operatorname{tr}\left(z_{1}\right)$ will trace a neighboring Jordan curve $\gamma_{t}$, as $z_{1}$ traces $\gamma$. Let $V$ with coordinate $z_{1}$ be the region interior to $\gamma_{t}$ and $U$ the region exterior to $\gamma$; the coordinate $z_{1}$ restricts to a neighborhood of the exterior of $\gamma$; call the restriction $z_{0}$. A deformed surface $R_{t}$ is defined by identifying the boundary of $U$ and the boundary of $V_{t}: p \in \gamma=\partial U$ is identified with the point $q \in \gamma_{t}=\partial V$ provided $z_{1}(q)=f\left(z_{0}(p), t\right)$ and $R_{t}=U \cup V / \sim$.

As given the identification is by sewing two regions along their boundaries. We would rather have an identification by overlapping open sets. Consider the image of $\bar{U}$ in $R_{t}$ with coordinate $z_{0}$ near $\gamma$ and the image of $\bar{V}$ in $R_{t}$ with coordinate $z_{1} ; z_{0}$ has an analytic continuation to a neighborhood of $\gamma=\partial U \subset R_{t}$ and $z_{1}$ an analytic continuation to a neighborhood of $\gamma_{t}=\partial \bar{V} \subset R_{t}$. Since $z_{1}=f\left(z_{0}, t\right)$ on $\gamma$ (a uniqueness set) it follows that the analytic continuations satisfy the same equation on a neighborhood of $\gamma$. In brief: we would obtain the same surface $R_{t}$ if $U$ and $V$ were enlarged (same notation) by including neighborhoods of their boundaries and the identification was by overlapping.

Now to give the 1-cocycle for the infinitesimal deformation. The context is just as in the previous section; the 1-cocycle for the Schiffer variation is

$$
\theta(t)=\frac{\partial f\left(z_{0}, t\right)}{\partial t} \frac{\partial}{\partial z_{1}}=r\left(z_{0}\right) \frac{\partial}{\partial z_{1}}=\frac{r\left(z_{0}\right)}{1+t r^{\prime}\left(z_{0}\right)} \frac{\partial}{\partial z_{0}}
$$

on $U \cap V$ giving a class in $\check{H}^{1}\left(R_{t}, \mathcal{O}\left(\kappa^{-1}\right)\right)$. Can the deformation be trivial? Recall that the pairing of $(\theta)$ with $\phi \in \check{H}^{0}\left(R, \mathcal{O}\left(\kappa^{\otimes 2} \zeta_{p_{1}} \cdots \zeta_{p_{n}}\right)\right)$ is simply the integral

$$
\int_{\gamma \approx \partial U} \frac{r\left(z_{0}\right)}{1+t r^{\prime}\left(z_{0}\right)} \phi
$$

(the integral is calculated in the variable $z_{0}$ ). We point out a few of the interesting cases. Let $t=0$ and $r$ be meromorphic in the interior of $\gamma$. The residue theorem can be applied; the integral is given by the sum of the residues of $r \phi$ interior to $\gamma$. We consider two specific cases. The first is for $r$ having a single simple pole, say at $q$, interior to $\gamma$. By Riemann-Roch $R$ has a holomorphic quadratic differential $\phi_{0}$ which is nonzero at $q$. The residue of $r \phi_{0}$ is nonzero and thus $r$ gives a nontrivial infinitesimal deformation. The second is for one of the distinguished points of $R$, say $p_{1}$, lying interior to $\gamma$. By Riemann-Roch $R$ has a meromorphic quadratic differential with its only pole simple and at $p_{1}$. Now if $r$ is holomorphic 
on the interior of $\gamma$ and nonvanishing at $p_{1}$ then the infinitesimal deformation is nontrivial. Of course this last deformation is simply sliding a point over a fixed surface. We would like to emphasize a particular choice of $r$ for the sliding deformation.

Definition. Let $\delta z_{0}\left(p_{1}\right)$ denote the infinitesimal Schiffer variation given by $r\left(z_{0}\right) \equiv 1$.

\section{Pinching a collar}

Consider an annulus $A$ in a surface $R$, situated such that a core curve $\gamma$ of $A$ represents a nontrivial free homotopy class in $R$. Let $h$ be a biholomorphism mapping $A$ to the circular annulus $\mathcal{A}=\left\{r<\left|z_{0}\right|<1\right\}$ in the $z_{0}$-plane. We recall how the twist-stretch deformation of $\mathcal{A}$ defines a deformation of $R$. To this end consider $z_{0}=h(p)$ and $z_{1}=1 / h(p)$ as coordinates on $A$. Choose $U$ and $V$ open in $R$, such that $U \cup V$ is a neighborhood of $A$ and $U \cap V=A$. Let $\left\{U_{a}\right\}$ be an atlas for $R$ such that each $U_{a}$ is either $U, V$ or disjoint from $A$. We can define a deformation of $R$ by simply redefining the overlap from $U$ to $V$. Specifically given $t$ near 1 , the new identification of $U$ to $V$ is given by defining for $u \in U$, $v \in V, u \sim_{t} v$ if $z_{0}(u) z_{1}(v)=t$. Denote the deformed surface by $R_{t}$. From Section 1 the 1 -cocycle (the map is $z_{1}=f\left(z_{0}, t\right)=t / z_{0}$ ) for the deformation is

$$
\theta=\frac{1}{z_{0}} \frac{\partial}{\partial z_{1}}=\frac{z_{1}}{t} \frac{\partial}{\partial z_{1}} \quad \text { on the overlap of } U \text { and } V .
$$

And for a quadratic differential $\phi$, holomorphic in $A$, the pairing $((\theta), \phi)$ is the period

$$
\frac{1}{t} \int_{\gamma} z_{1} \phi
$$

The literature for the pinching deformation is extensive. We only cite a few open questions. Evidently it is not known (without some hypothesis on $h$ ) if the infinitesimal deformation is in general nontrivial. One can also consider the following: choose $3 g-3, g$ the genus of $R$, disjoint annuli $A_{1}, \ldots, A_{3 g-3}$ in $R$ and charts $h_{j}: A_{j} \rightarrow \mathbf{C}$ such that each component of $R-\bigcup_{j} A_{j}$ is topologically $S^{2}-\{3$ points $\}$. Does the family $R\left(s_{1}, \ldots, s_{3 g-3}\right)=R_{t_{1}, \ldots, t_{3 g-3}}$ for $t_{j}=e^{s_{j}}$ formed by pinching the collars, map injectively to the Teichmüller space of $R$ ? And similarly given an infinitesimal deformation of $R$ and the free homotopy class of a simple closed curve, does there exist an annulus $A$, representing the free homotopy class, and chart $h: A \rightarrow \mathbf{C}$, such that the infinitesimal pinching is the specified deformation? We expect the answer to be yes; in the next section we shall examine the analogous question for opening a node. 


\section{Plumbing a node}

The family for pinching a collar can be extended by allowing the parameter to tend to zero. The result is a family of Riemann surfaces over the punctured disc. We shall consider the effect of completing the family by including a special fibre, a Riemann surface with nodes.

We start with an example. Consider the germ $V=\{z w=t|| z|| w,|| t \mid,<1\}$ $\subset \mathbf{C}^{3}$ of a variety. The defining function is $z w-t$ with differential $z d w+w d z-d t$; as a consequence $V$ is smooth with global coordinate $(z, w)$. The projection $\Pi(z, w)=z w=t$ maps $V$ to the $t$ unit-disc $D=\{|t|<1\}$. The differential $d \Pi=z d w+w d z$ vanishes only at the origin; $\Pi$ is almost a fibration. What are the fibres? For $t \neq 0$ the fibre is the annulus $\{|t|<|z|<1, w=t / z\}$ and the 0 -fibre is the intersection of the unit ball in $\mathbf{C}^{2}$ with the union of the coordinate axes. Removing the special point, the origin, (a node) the union becomes $\{0<|z|<1\}$ $\cup\{0<|w|<1\}$. In fact $\Pi: V \rightarrow D$ is the fundamental example of a family of Riemann surfaces with nodes; note that $V$ and $D$ are smooth $\mathbf{C}$-manifolds, $\Pi$ is holomorphic, and a node is the germ of the coordinate axes in $\mathbf{C}^{2}$.

A Riemann surface with nodes $R$ is a connected complex space, such that each point has a neighborhood isomorphic to either the unit disc in $\mathbf{C}$ or the germ of the coordinate axes in $\mathbf{C}^{2}$. We will tacitly assume that $R$ is compact, and that each component of $R_{0}=R-$ \{nodes\} has a negative Euler characteristic. Such an $R$ is a stable curve in the sense of Mumford, [DMu, Mu]. A degenerating family $\Pi: M \rightarrow B$ of compact Riemann surfaces is a proper holomorphic map $\Pi$ of smooth complex manifolds with generic fibre a compact Riemann surface. A special fibre may have a finite number of nodes, points where the local model for the fibration is the above example. On removing a node $n \in R$ we obtain a pair of punctures $a$ and $b$ of $R-\{n\}$; we will refer to $a$ and $b$ as being paired to form $n$. A degenerating family $\Pi: M \rightarrow B$ defines a holomorphic map of the base $B$ to $\overline{\mathcal{M}_{g}}$, the Deligne-Mumford compactification of the classical moduli space $\mathcal{M}_{g}$ of Riemann surfaces, [DMu, Mu]. We shall use the description of $\overline{\mathcal{M}_{g}}$ given in the work of Bers [Be], Earle-Marden [EMr], Fay [F], and Masur [Ms]. A further exposition of their description is given in [W]. In all cases the local geometry of $\overline{\mathcal{M}_{g}}$ is analyzed by giving constructions of specific families.

The standard construction to include a noded surface in a degenerating family is plumbing. Suppose for simplicity that $R$ has a single node $n . R_{0}=R-\{n\}$ is a smooth noncompact surface with punctures $a$ and $b$. Choose a coordinate $u$ for a neighborhood $U$ of $a$ with $u(a)=0$ and a coordinate $v$ for a neighborhood $V$ of $b$ with $v(b)=0$. Given $c>0$ consider the open surface $R_{*}=R_{0}-\{|u|<c\}$ $\cup\{|v|<c\}$, the germ $\mathcal{V}=\left\{z w=t|| z|| w,|<c| t \mid,<c^{2}\right\}$ and the disc $D=$ $\left\{|t|<c^{2}\right\}$. We define a map $f(p, t)$ from $(U \cup V) \times D$ to $\mathcal{V}$

$$
\begin{array}{ll}
(p, t) \rightarrow(u(p), t / u(p), t) & \text { for } \quad p \in U \subset R_{*}, \\
(p, t) \rightarrow(t / v(p), v(p), t) & \text { for } \quad p \in V \subset R_{*} .
\end{array}
$$


The map generates an equivalence relation $\simeq_{f}$ on $\left(R_{*} \times D\right) \cup \mathcal{V}$; the identification space $\operatorname{Plumb}_{R}(u, v)=\left(R_{*} \times D\right) \cup \mathcal{V} / \simeq_{f}$ is a degenerating family with projection to $D$, and 0 -fibre the original noded surface $R$. The data for the family is the tuple $(R, U, u, V, v, c)$; in practise $U, V$ are understood and $c$ is fixed beforehand. The reader can check that the family is independent of $c$ for $t$ small. As noted above the family gives a holomorphic map of $D$ to $\overline{\mathcal{M}_{g}}: 0$ maps to a point in the compactification divisor of $\overline{\mathcal{M}_{g}}$ and $t \neq 0$ maps to a point of $\mathcal{M}_{g}$.

We would like to find how the initial tangent of a plumbing depends on the choice of the coordinates $u$ and $v$. Can an arbitrary infinitesimal deformation opening the node of a noded surface be realized by plumbing? Obviously such a calculation will require a model for the tangent and cotangent spaces of $\overline{\mathcal{M}_{g}}$ at a noded surface. A description suited for our purposes is contained in the paper of Masur, [Ms]. Specifically the cotangent bundle (a $V$-bundle over a $V$-manifold) is described as a modification of the bundle of regular quadratic differentials for a degenerating family. Assume for simplicity that an initial family $\Pi: M \rightarrow P D$ is given as follows: $P D$ is a polydisc in $s$-space, $\Pi^{-1}(s)=R_{s}$ is a smooth surface with two punctures $a_{s}$ and $b_{s}$ (equivalently $R_{s}$ is smooth compact and $a_{s}, b_{s}$ are disjoint sections). It will be simpler if we only consider the general case: if $R_{s}$ has genus $g>0$ assume $P D$ has dimension $3 g-1$ and the induced map of $P D(P D /\{$ symmetries $\})$ to $\mathcal{M}_{g, 2}$ is injective. Let $u, v$ give rise to holomorphic charts about the punctures: $(u, \Pi): U \rightarrow \mathbf{C} \times P D, U$ a neighborhood in $M$ of $a_{s}$, $u\left(a_{s}\right)=0$ and $(v, \Pi): V \rightarrow \mathbf{C} \times P D, V$ a neighborhood in $M$ of $b_{s}, v\left(b_{s}\right)=0$ (trivializations of neighborhoods of the sections). Now we describe how to plumb each fibre of $\Pi: M \rightarrow P D$ ( $a_{s}$ and $b_{s}$ are first identified to form a noded family). Arrange, restricting the $s$-domain if necessary, that the range of $u$ and $v$ each contain the polydisc $\{|\zeta|<c\} \times P D, c>0$. Start with the family of open surfaces $M_{*}=M-\{|u|<c\} \cup\{|v|<c\}$, the germ $\mathcal{V}=\left\{z w=t|| z|| w,|<c| t \mid,<c^{2}\right\}$, and the disc $D=\left\{|t|<c^{2}\right\}$. Define a map $h(p, t)$ from $(U \cup V) \times D$ to $P D \times \mathcal{V}$

$$
\begin{array}{ll}
(p, t) \rightarrow(\Pi(p), u(p), t / u(p), t) & \text { for } \quad p \in U \subset M_{*}, \\
(p, t) \rightarrow(\Pi(p), t / v(p), v(p), t) & \text { for } \quad p \in V \subset M_{*} .
\end{array}
$$

The map generates an equivalence relation $\simeq_{h}$ on $\left(M_{*} \times D\right) \cup(P D \times \mathcal{V})$; the identification space $\operatorname{Plumb}_{M}(u, v)$ is a degenerating family with projection $\Pi_{0}$ to $P D \times D$. By definition the $\mathcal{V}$-collar will be the intersection of $P D \times \mathcal{V} \subset$ Plumb $_{M}(u, v)$ and a fibre of $\Pi_{0}^{-1}$. By our hypothesis on $\Pi: M \rightarrow P D$ the induced map of $P D \times D$ to $\overline{\mathcal{M}_{\gamma}}, \gamma=g+1$ provides a local coordinate chart $\left(s_{1}, \ldots, s_{n}, t\right)$, $n=3 g-1$. We require one last definition: $Q D$ the space of regular quadratic differentials (in the sense of Bers, equivalently the pushdown of the square of the relative dualizing sheaf). For a smooth surface $S$ the space $Q D(S)$ is simply $\check{H}^{0}\left(S, \mathcal{O}\left(\kappa^{\otimes 2}\right)\right)$ the holomorphic quadratic differentials on $S$. For a noded surface $R$ then $Q D(R)$ is the vector space of meromorphic quadratic diffentials $\phi$ on 
$R_{0}=R-\{$ nodes $\}$ such that : each $\phi$ has poles only at the punctures of $R_{0}$, the poles have order at most 2 , and for paired punctures $a$ and $b$, then $\operatorname{Res}_{a} \phi=$ $\operatorname{Res}_{b} \phi$. By Riemann-Roch each fibre of $Q D$ has rank $3 \gamma-3$. We are ready to state Masur's result.

Theorem [Ms, expansion 5.3 and Proposition 7.1]. Let $\gamma$ be the genus of the generic fibre of the family $\operatorname{Plumb}_{M}(u, v)$, there exist functions $a_{1}, \ldots, a_{3 \gamma-3}$ such that:

i) $a_{j}(z, s, t)$ is holomorphic on $\{|t| / c<|z|<c\} \times P D \times D$,

ii) $a_{j}(z, s, t)(d z / z)^{2}$ is the restriction to the $\mathcal{V}$-collar in $R_{s, t}=\Pi_{0}^{-1}(s, t)$ of a regular quadratic differential $\phi_{j}$ of $R_{s, t}$,

iii) $\left\{\phi_{j}\right\}$ form a basis for $Q D\left(R_{s, t}\right)$,

iv) for the local coordinate $\left(s_{1}, \ldots, s_{n}, t\right)$ on $\overline{\mathcal{M}_{\gamma}} d s_{1}=\phi_{1}, \ldots, d s_{n}=\phi_{n}$, $d t=t / 2 \pi i \phi_{n+1}$ (in the sense of linear functionals acting on the infinitesimal deformations).

Statement iv) provides a local model for the cotangent bundle of $\overline{\mathcal{M}_{\gamma}}$. As a sample calculation, consider varying $t$ for the $\mathcal{V}$-collar $\{z w=t\}$ in $R_{s, t}$. The infinitesimal deformation is simply the period for pinching a collar, for $\phi \in Q D$

$$
\left(\frac{\partial}{\partial t}, \phi\right)=\frac{1}{t} \int_{\alpha} z \phi
$$

where $\alpha$ is a core curve for the collar. Evaluating for the basis $\left\{\phi_{j}\right\}$ we find the constant terms for the Laurent expansion in $z: a_{n+1}$ has constant term $1, a_{j}$, $j \neq n+1$, has constant term zero. This agrees with Masur's formulas (note that we have suppressed a factor of $i / 2$ from the pairing for Serre duality).

We would like to start our analysis by giving a construction to compare the families $\operatorname{Plumb}_{R}(u, v)$ and $\operatorname{Plumb}_{R}(u, G(v))$, where $R$ is a surface with a single node $n, R-\{n\}$ has punctures $a$ and $b$, and $G$ is the germ at $0 \in \mathbf{C}$ of a biholomorphism with $G(0)=0$. The essential matter is to relate the families $u v=t$ and $u G(v)=\tau$. As notation let $\partial / \partial t$ be the initial $\operatorname{Plumb}_{R}(u, v)$ tangent, $\partial / \partial \tau$ the initial Plumb $_{R}(u, G(v))$ tangent and $\delta u(a)$ the infinitesimal sliding of $a$ in the $u$-coordinate on $R$ (see end of Section 2).

Lemma. With the above notation $\partial / \partial \tau=\left(G^{\prime}(0)\right)^{-1}\left((\partial / \partial \tau)+\frac{1}{2} G^{\prime \prime}(0) \delta u(a)\right)$.

Proof. The first matter is to check the scaling of $\tau$. For $\lambda=\tau / G^{\prime}(0)$ the families for $u G(v)=\tau$ and $u\left(G(v) / G^{\prime}(0)\right)=\lambda$ coincide; thus $\partial / \partial \tau=$ $\left(G^{\prime}(0)\right)^{-1}(\partial / \partial \lambda)$ and for the remaining discussion we can assume $G^{\prime}(0)=1$. As a technical matter we choose $c$ such that the restriction of $G$ to $\{|v|<2 c\}$ is a biholomorphism, sufficiently close to the identity map. And for latter reference we define a nonlinear transform of $G$,

$$
\langle G\rangle=\frac{G(y)-y G^{\prime}(y)}{y^{2} G^{\prime}(y)} ;
$$


$\langle G\rangle$ is a holomorphic germ at the origin with $\langle G\rangle(0)=-\frac{1}{2} G^{\prime \prime}(0)$.

Start by considering the coordinate $z$ of $\mathcal{V}$ : for a $\mathbf{C}$-parameter $\varepsilon,|\varepsilon|<c^{2}$, define a new coordinate $z_{*}$ by

$$
z_{*} G(\varepsilon / z)=\varepsilon
$$

(i.e. for $z$ in a neighborhood of $|z|=c$ define $z_{*}$ with domain $N$ a neigborhood of $\left.\left|z_{*}\right|=c\right)$. Now we construct a family $S p a n$ which includes both $\operatorname{Plumb}_{R}(u, v)$ and $\operatorname{Plumb}_{R}(u, G(v))$. Start with $R_{*}=R_{0}-\{|u|<c\} \cup\{|v|<c\}, D=\left\{|t|<c^{2}\right\}$, $\mathcal{V}=\{z w=t\}, \Delta=\left\{|\varepsilon|<c^{2}\right\}$ and $N=$ (neighborhood of $\left|z_{*}\right|=c$ ). The identification of $R_{*} \times D \times \Delta$ to $N \times D \times \Delta$ is given by the map

$$
(p, t, \varepsilon) \rightarrow(u(p), t, \varepsilon) \quad \text { for } \quad p \in U \subset R_{*},
$$

the identification of $N \times D \times \Delta$ to $\mathcal{V} \times \Delta$ is given by the map

$$
\left(z_{*}, t, \varepsilon\right) \rightarrow\left(z=\varepsilon / G^{-1}\left(\varepsilon / z_{*}\right), w=t G^{-1}\left(\varepsilon / z_{*}\right) / \varepsilon, t, \varepsilon\right)
$$

and the $R_{*} \times D \times \Delta$ to $\mathcal{V} \times \Delta$ identification is unchanged

$$
(p, t, \varepsilon) \rightarrow(t / v(p), v(p), t, \varepsilon) \quad \text { for } \quad p \in V \subset R_{*} .
$$

We have the following picture for $t$ and $\varepsilon$ fixed.

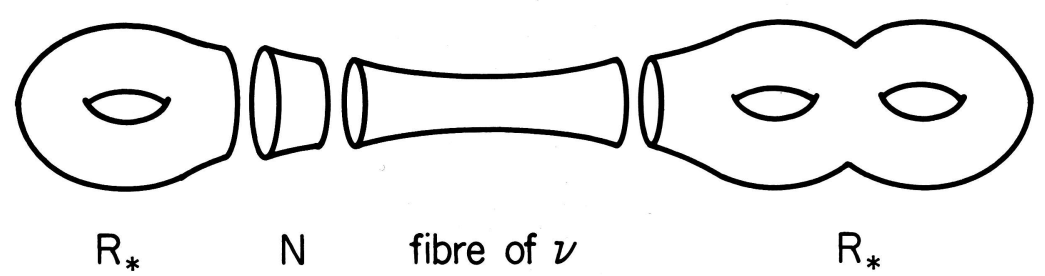

Form the identification space Span of $R_{*} \times D \times \Delta, N \times D \times \Delta$, and $\mathcal{V} \times \Delta$; Span is a family over $D \times \Delta$. The next item is to check for the subfamilies. For $\varepsilon=0$ then $z_{*}=z$ and the identification of $U \subset R_{*}$ to a fibre of $\mathcal{V}$ is simply that of $\operatorname{Plumb}_{R}(u, v)$. In brief, the restriction of Span to $D \times\{\varepsilon=0\}$ is $\operatorname{Plumb}_{R}(u, v)$. Now consider the restriction of Span to the diagonal $\{(t, \varepsilon=t)\} \subset D \times \Delta$. Let us concentrate on the identification of a fibre of $N \times D \times \Delta$ to an open set in a fibre of $\mathcal{V} \times \Delta$. Fix $t \neq 0$, set $\varepsilon=t$; the identification is given by $z_{*}=t / G(t / z)$ for $\left|z_{*}\right| \approx c$. The sequence of identifications $R_{*} \times D \times \Delta$ to $N \times D \times \Delta$ and $N \times D \times \Delta$ to $\mathcal{V} \times \Delta$ is now equivalent to identifying $R_{*} \times D \times \Delta$ to $\mathcal{V} \times \Delta$ by the map

$$
(p, t, t) \rightarrow\left(z=t / G^{-1}(t / u(p)), w=G^{-1}(t / u(p)), t, t\right) .
$$


The identification $w=G^{-1}(t / u(p))$ or, equivalently $u(p)=t / G(w)$, provides for the analytic continuation $\tilde{u}$ of $u$ to the entire $\mathcal{V}$-fibre $=\{|t| / c<|w|<|c|$, $z=t / c\}$. The identification of the $\mathcal{V}$-fibre and $V \subset R_{*}$ is given by $w=v(p)$; substituting we find the equation $\tilde{u}=t / G(v)$ or $\tilde{u} G(v)=t$. If we perform the analogous analytic continuation of $u$ across the $\mathcal{V}$-collar in the $t$-fibre of $\operatorname{Plumb}_{R}(u, G(v))$ we find the same equation; the families $\left.S p a n\right|_{\text {diagonal }}$ and $\operatorname{Plumb}_{R}(u, G(v))$ coincide.

Span is a family in $\varepsilon$ and $t$. The 1 -cocycle for the $\varepsilon$-variation is (the map is $\left.z_{*}=\varepsilon / G(\varepsilon / z)\right)$

$$
\Omega(N \times D \times \Delta, \mathcal{V} \times \Delta)=\frac{G(\varepsilon / z)-G^{\prime}(\varepsilon / z) \varepsilon / z}{G(\varepsilon / z)^{2}} \frac{\partial}{\partial z_{*}}=\langle G\rangle(\varepsilon / z) \frac{\partial}{\partial z}
$$

and the 1-cocycle for the $t$-variation is an infinitesimal pinching

$$
\theta=\frac{z}{t} \frac{\partial}{\partial z} \quad \text { on an overlap in } \mathcal{V} \times \Delta .
$$

The family $\mathrm{Plumb}_{R}(u, G(v))$ occurs as the restriction of Span to $\{(t, t)\} \subset D \times \Delta$ and thus the tangent field of $\operatorname{Plumb}_{R}(u, G(v))$ is the restriction of $(\partial / \partial t+\partial / \partial \varepsilon)$ to $\{(t, t)\}$. Now the pairing of $(\partial / \partial t+\partial / \partial \varepsilon)$ with $\phi \in Q D$ is the period

$$
\int_{|z|=c}\left(\frac{z}{t}+\langle G\rangle\left(\frac{\varepsilon}{z}\right)\right) \phi
$$

and the value on the diagonal is given by setting $\varepsilon=t$. As the final step consider that Span is embedded in a family as for Masur's result. We wish to find the limit of the period as $t \rightarrow 0$. By the theorem the elements of $Q D$ converge uniformly on $|z|=c$; the limit of the period $\int_{|z|=c}\langle G\rangle(t / z) \phi$ is simply $-\frac{1}{2} G^{\prime \prime}(0) \int_{|z|=c} \phi$. In summary: the initial tangent of Span $\left.\right|_{\text {diagonal }}$ is $(\partial / \partial t)+\frac{1}{2} G^{\prime \prime}(0) \delta u(a)$ for $\partial / \partial t$ the tangent relative to the coordinate $\left(s_{1}, \ldots, s_{n}, t\right)$ on $\overline{\mathcal{M}_{\gamma}}$, and $\delta u(a)$ the infinitesimal sliding. The proof is complete.

Let $\partial / \partial \sigma$ now be the initial tangent of the family $\operatorname{Plumb}_{R}(F(u), G(v))$ for $F$ a germ at $0 \in \mathbf{C}$ of a biholomorphism.

Corollary. With the above notation

$$
\frac{\partial}{\partial \sigma}=\left(G^{\prime}(0) F^{\prime}(0)\right)^{-1}\left(\frac{\partial}{\partial t}+\frac{1}{2} G^{\prime \prime}(0) \delta u(a)+\frac{1}{2} F^{\prime \prime}(0) \delta v(b)\right) .
$$

Proof. Apply the lemma to $\operatorname{Plumb}_{R}(u, G(v))$ and $\operatorname{Plumb}_{R}(F(u), G(v))$. The conclusion is

$$
\frac{\partial}{\partial \sigma}=\left(F^{\prime}(0)\right)^{-1}\left(\frac{\partial}{\partial \tau}+\frac{1}{2} F^{\prime \prime}(0) \delta G(v)(b)\right)
$$


By Section 2 the infinitesimal sliding satisfies the transformation law $\delta G(v)(b)=$ $\left(G^{\prime}(0)\right)^{-1} \delta v(b)$. Apply the lemma a second time to give $\partial / \partial \tau$ in terms of $\partial / \partial t$ and $\delta u(a)$. The conclusion follows.

Remarks. Our approach is based on a local construction for the plumbing collars and the description of $Q D$. The method could also be used to establish the analogous result for the case of a surface with several nodes. The final result shows that by varying $F$ and $G$ the initial tangent of $\operatorname{Plumb}_{R}(F(u), G(v))$ in $\overline{\mathcal{M}_{\gamma}}$ varies in the three dimensional subspace $T P=\operatorname{span}\{(\partial / \partial t), \delta u(a), \delta v(b)\}$ (note: $T P$ has dimension $=1+\operatorname{dim} \operatorname{span}\{\delta u(a), \delta v(b)\} \subset$ infinitesimal deformations of $R$-\{node\}. In fact $F$ and $G$ can be chosen to realize an arbitrary vector of $T P$ with nonzero $\partial / \partial t$ component; $T P$ defines a rank three subbundle (the span of all possible initial plumbing tangents) of the tangent bundle of $\overline{\mathcal{M}_{\gamma}}$, restricted to the locus of stable curves with a single node. We could also consider the codimension three subbundle of cotangent vectors annihilated by TP. By Masur's theorem and the discussion of the tangent-cotangent pairing, the annihilated subspace corresponds to the regular quadratic differentials, holomorphic on the compactification of $R-$ \{node\}. And finally we note that in order to obtain the expansion of a quantity on $\overline{\mathcal{M}}_{\gamma}$ at a noded curve, it is necessary to consider more than the plumbing construction. 


\section{References}

[Ah] Ahlfors, L.V.: Some remarks on Teichmüller's space of Riemann surfaces. - Ann. of Math. 74, 1961, 171-191.

[Be] Bers, L.: Spaces of degenerating Riemann surfaces. - Ann. of Math. Stud. 79, 1974, 43-55.

[DMu] Deligne, P., and D. Mumford: The irreducibility of the space of curves of given genus. - Inst. Hautes Études Sci. Publ. Math. 36, 1979, 75-110.

[EMr] Earle, C., and A. Marden: Geometric complex coordinates for Teichmüller space. Manuscript.

[F] FAY, J.D.: Theta functions on Riemann surfaces. - Lecture Notes in Mathematics 352. Springer-Verlag, New York-Heidelberg, 1973.

[G] Gunning, R.C.: Lectures on Riemann surfaces. - Princeton University Press, Princeton, N. J., 1966.

[Ms] Masur, H.: The extension of the Weil-Petersson metric to the boundary of Teichmüller space. - Duke Math. J. 43, 1976, 623-635.

[MwK] Morrow, J., and K. Kodaira: Complex manifolds. - Holt, Rinehart and Winston, New York, 1971.

[Mu] Mumford, D.: Stability of projective varieties. - Enseign. Math. (2) 23, 1977, 39-110.

[ScS] Schiffer, M., and D.C. SPEnCER: Functionals of finite Riemann surfaces. - Princeton University Press, Princeton, N. J., 1954.

[W] Wolpert, S.A.: The hyperbolic metric and the geometry of the universal curve. - J. Diff. Geom. (to appear).

University of Maryland

Department of Mathematics

College Park, MD 20742

U.S.A.

Received 3 March 1988 